\title{
Percepción de la intensidad al esfuerzo: Un estudio multi-method en actividad física
}

\author{
Perception of the intensity of the effort:
} A multi-method study on physical activity

\author{
Percepção da intensidade do esforço: \\ Um estudo multi-method em actividade física
}

\author{
Marta Castañer ${ }^{1 *}$, Glòria Saüch¹, Oleguer Camerino', Pedro Sánchez-Algarra² y M. Teresa Anguera ${ }^{3}$ \\ ${ }^{1}$ Laboratorio de Observación de la Motricidad. Instituto Nacional de Educación Fisca de Catalunya-(INEFC)-Universidad de Lleida (España), \\ ${ }^{2}$ Facultad de Biologia-Universidad de Barcelona (España), ${ }^{3}$ Facultad de Psicología-Universidad de Barcelona (España)
}

Resumen: Este estudio aborda un doble objetivo: a) conocer si existe progresión significativa entre tres valores correspondientes al índice de Borg elicitados por jóvenes deportistas con relación a tres actividades de intensidad creciente; b) analizar la relación existente entre el índice de masa corporal (IMC) de los participantes con los valores de frecuencia cardíaca (FC) y la percepción de intensidad del esfuerzo (RPE). Método: Treinta y cinco deportistas jóvenes con una edad media de 18 y 28 ańos $(21 \pm 0,5)$, realizaron 3 actividades que exigían una intensidad creciente de ejercicio: leve, moderada y vigorosa (ACSM, 2011). Se controló la frecuencia cardíaca mediante el dispositivo telemétrico Polar Team 2 y al finalizar las tres actividades se administró tres veces la escala de Borg para obtener la RPE para cada actividad. Se ha complementado la metodología observacional indirecta (índices de Borg) con la metodología cuasiexperimental para obtener inferencias causales mediante un diseño sincrónico de variable de asignación conocida y grupo único. Se ha realizado un análisis de regresión múltiple, considerando la FC y la RPE como variables predictoras del IMC. Resultados: la $\mathrm{RPE}$ es creciente, acorde a las actividades, en todos los participantes, aunque en ningún caso la tendencia es estadísticamente significativa. Prácticamente la cuarta parte del IMC se puede explicar por la FC y la RPE puesto que los participantes que tienen valores más altos de FC y de índice de Borg tienen también mayores valores de IMC. Este estudio puede proporcionar una visión de evaluación integrada entre los factores IMC, FC y la RPE, que suelen investigarse de modo aislado.

Palabras clave: Intensidad del ejercicio físico, tasa cardíaca, índice de masa corporal, regresión múltiple, regresión simple.

Abstract: The current study had two aims: a) to determine whether the three Borg scale values assigned by young athletes to three activities of increasing intensity showed a significant progression; and b) to analyse the relationship between the body mass index (BMI) of these athletes and their heart rate (HR) and rating of perceived exertion (RPE). Method: Thirtyfive young athletes (mean age 21 years \pm 0.5 ) performed three activities involving an increasing intensity of exercise: mild, moderate and vigorous (ACSM, 2011). Heart rate was monitored using the Polar Team 2 system, and at the end of each activity participants completed the Borg RPE scale. Indirect observational methodology (Borg scale) was complemented by a quasi-experimental approach in order to make causal inferences by means

Dirección para correspondencia [Correspondence address]: Marta Castañer. INEFC. Partida de la Caparrella, s/n. 25110 Lleida (Espańa) http://lom.observesport.com / E-mail: mcastaner@inefc.es of a synchronous design involving a known assignment variable and a single group. A multiple regression analysis was performed, considering HR and RPE as predictors of BMI. Results: The RPE of all participants increased across the three activities, but in no case did the trend achieve statistical significance. Participants with higher values of HR and RPE also had a higher BMI, and in the regression analysis HR and RPE explained around one-quarter of BMI. This study provides an integrated assessment of the relationship between BMI, HR and RPE factors that are usually examined isolated.

Keywords: Exercise intensity, heart rate, body mass index, multiple regression, simple regression.

Resumo: $\mathrm{O}$ estudo teve um duplo objetivo: a) determinar se os três valores da escala de Borg atribuídos por jovens atletas, em três atividades de intensidade crescente, mostrou uma progressão significativa; b) analisar a relação entre o índice de massa corporal (IMC) destes atletas, a sua freqüência cardíaca (FC) e percepçáo subjetiva de esforço (RPE). Trinta e cinco atletas jovens (idade média de 21 anos $\pm 0,5$ ) realizaram três atividades que exigiam uma intensidade crescente de exercício: leve, moderada e vigorosa (ACSM, 2011)._A freqüência cardíaca foi monitorizada através do sistema Polar Team 2, e no final de cada atividade os participantes completaram a escala Borg para obter a RPE de cada atividade.

Complementou-se a metodología observacional indirecta (índices de Borg) com a metodología quase-experimental para obter inferências causais através de um desenho sincrónico de variável de atribuição conhecida e com grupo único. Foi realizada uma análise de regressão múltipla, considerando a FC e a RPE como variáveis preditoras do IMC. Resultados: a RPE é crescente, em concordância com as atividades, em todos os os participantes ainda que em nenhum caso a tendência seja estatisticamente significativa. Praticamente um quarto do IMC pode ser explicado pela FC e pela RPE pois os participantes que têm valores mais elevados de FC e de índice de Borg têm também valores mais elevados de IMC. Este estudo pode proporcionar uma visão de avaliação integrada entre os factores IMC, FC e a RPE, que podem investigar-se isoladamente

Palavras chave: Intensidade de exercício físico, frequência cardíaca, índice de massa corporal, regressão múltipla, regressão simples. 


\section{Introducción}

Las personas que se mantienen físicamente activas poseen un mejor estado de salud que aquellas que desarrollan hábitos sedentarios (Plasqui y Westerterp, 2007). La realización de escasa o poca actividad física está relacionada con diversas alteraciones psicofisiológicas, entre las que destacan especialmente, las enfermedades cardiovasculares y la obesidad (Browson, Boehmer y Luke, 2005; Fang, 2003). Estudiar la percepción de la intensidad del esfuerzo (RPE), la frecuencia cardíaca (FC) y el índice de masa corporal (IMC) en jóvenes deportistas como sector específico de la población, ofrece indicadores con relación a la práctica óptima de la actividad física y deportiva (Calahorro, Torres y Lara, 2014).

Los modelos de estilo de vida actuales incluyen unos estándares en los que cobran un especial interés los hábitos equilibrados (Saüch y Castañer, 2014), entre ellos la frecuencia, la intensidad y la duración de la práctica de la actividad física, en adelante PA, para ser afines a la nomenclatura internacional que usa la literatura científica (Ainsworth, 2000). Para poder planificar la PA se debe tener en cuenta la RPE (Borg, 1962) como asequible y sobretodo que contribuya a la mejora del bienestar físico, psíquico y social (Blair y Morris, 2009).

En la actualidad es importante el control del esfuerzo de la actividad física. Existen diferentes vías para medir la intensidad de esfuerzo a la que se someten los deportistas en sus modalidades deportivas específicas o participantes de diverso rango que siguen un programa de PA bien sea de perfil competitivo, utilitario, educativo o terapéutico (Castañer y Camerino, 2006; Castañer y Saüch, 2014; Castañer, Torrents, Anguera, Dinušová y Jonsson, 2009). La monitorización cardíaca se considera un método no invasivo de carga interna así como lo es la captación de valores del consumo de oxígeno $\left(\mathrm{VO}_{2}\right)$ a los cuales, a veces, se aplica de manera complementaria el análisis de concentración de ácido láctico en la sangre, este último considerado como método invasivo (CuadradoReyes, Chirosa, Chirosa, Martín y Aguilar, 2012).

Estos métodos permiten obtener parámetros objetivos del esfuerzo, pero la RPE a la hora de realizar un mismo ejercicio es una dimensión que no se puede obviar y cada vez más se está tomando en consideración tanto en estudios de esfuerzo (Naclerio, Barriopedro y Rodriguez, 2009, Lagally, McGraw, Young, Heathery Thomas, 2004) como en la implementación de programas de actividad física y deportiva (Calahorro et al., 2014; Gomez-Piriz, Jiménez- Reyes, Ruiz- Ruiz, 2011). Algunas investigaciones sostienen que el registro de la FC es un buen indicador para controlar la intensidad de la actividad física (Calahorro et al., 2014; Guerra, Chaves, Barros y Tirapegui, 2004; Reaburn y Abt, 2003). La escala de Borg estandarizada ad hoc para obtención de la RPE es un método no invasivo y sin coste económico de fácil aplicación en las sesiones de PA, y especialmente en competiciones y entrenamientos deportivos. Dicha escala constaba, en un inicio, de una valoración de 0 a 15 puntos y posteriormente fue modificada de 0 a 10 puntos (Borg, 1982). Se han realizado diversos estudios para comprobar la validez de dicha escala (Chen, Fan y Moe, 2002), en entrenamientos de balonmano (Fernández-Castanys, Chirosa y Chirosa, 2002; Bar-Or, Skinner, Bruskirky y Borg, 1972; Borg y Linderholm, 1970). Es la escala más utilizada para medición del esfuerzo en deportes individuales (Impellizzeri, Rampinini, Coutts, Sassi y Marcora, 2004) y colectivos tales como el fútbol (Alexiou y Coutts, 2008; Casamichana, Castellano y Blanco-Villaseñor, 2012) y el rugby (Hartwig, Naughton y Searl, 2008). Se considera una escala también adecuada para poder cuantificar y programar la intensidad de las sesiones y ya existe literatura que ha corroborado la correlación existente entre la FC y la RPE (Impellizzeri et al., 2004; Cuadrado-Reyes et al., 2012). El IMC o índice de Quetelet es un indicador de evaluación antropométrica altamente tenido en cuenta en el ámbito de la PA y el deporte que correlacionaremos en este estudio desde una perspectiva novedosa, puesto que se suele considerar como una variable a considerar para reforzar otras variables o datos pero no como un indicador directamente afectado por otros parámetros.

Los objetivos de este trabajo recaen en: a) conocer si existe progresión significativa entre tres valores del índice de Borg a partir de las tres respectivas respuestas elicitadas de los participantes a tres tipos de PA de creciente intensidad; b) analizar la relación existente entre el IMC de jóvenes deportistas con los valores de FC i RPE.

\section{Método}

\section{Diseño}

Desde una perspectiva multi-method (Anguera, Camerino, Castañer, y Sánchez-Algarra, 2014), se han complementado la metodología observacional indirecta (necesaria para obtener los índices de Borg) y la metodología cuasiexperimental, con el fin de obtener inferencias causales mediante un diseño sincrónico de variable de asignación conocida y grupo único.

\section{Participantes}

Han participado un total de 35 jóvenes deportistas, $8 \mathrm{mu}$ jeres y 27 hombres, de edades comprendidas entre 18 y 28 años $(21 \pm 0,5)$. La muestra se ha obtenido mediante muestreo aleatorio simple de un total de 120 alumnos que cursaban el primer cuatrimestre académico del grado de Ciencias de la Actividad Física del Deporte del Instituto Nacional de Educación Física de la Universidad de Lérida. Son jóvenes depor- 
tistas con una media de dedicación semanal de práctica de PA de 3 $\pm 1,4$ horas semanales. Para determinar los criterios de exclusión preguntamos a los alumnos si tomaban alguna medicación relacionada con algún tratamiento agudo o crónico, y así evitar un sesgo en los parámetros de FC. Todos ellos fueron informados del estudio que se iba a realizar y todos dieron su consentimiento informado.

\section{Instrumentos}

Mediante báscula y medidor de altura se obtuvieron los datos correspondientes al peso y la talla de los participantes, necesarios para obtener el IMC. Se utilizó la escala de Borg (1982) que despliega los rangos del 0 al 10 para valorar la RPE de los participantes. La FC se obtuvo mediante el dispositivo telemétrico Polar Team 2 v. 1.4.5.

\section{Procedimiento}

Dos profesores expertos (Dreyfus y Dreyfus, 1986; Genberg, 1992) en PA diseñaron una sesión ad hoc de nueve minutos, en la que se realizaban 3 actividades que exigían una intensidad creciente de ejercicio: leve, moderada y vigorosa según los rangos que indica el American College of Sport Medicine, 2011). El grupo de participantes se dividió en 5 microgrupos a razón de diez participantes por grupo. A todos se les controló la FC, segundo a segundo, mediante monitorización continua individualizada y almacenamiento de datos del equipo Polar Team. Al inicio de la intervención se activó el dispositivo y se paró al finalizar la sesión; este procedimiento se repitió de la misma manera con todos los grupos.

Una vez finalizada la sesión se llevó a cabo la fase de observación indirecta que, tras desinstalarles los Polars Teams consistíó en concentrar a los participantes para llevar a cabo una conversación de rememoración de las tres actividades realizadas. Al tratarse de jóvenes deportistas, conocen el funcionamiento de la escala de Borg por experiencias previas, pero esta fase de observación indirecta permitió una puesta en común conjunta de todos los participantes acerca de cómo valorar la RPE para cada una las tres actividades realizadas, sobre las puntuaciones del 0 al 10 de dicha escala. Esta fase de observación indirecta permitió establecer elicitaciones de respuesta individual de cada participante mediante una pequeña entrevista acerca de datos de género, edad, altura y peso corporal así como la tipología de deporte que practica habitualmente y el conocimiento de sus hábitos de práctica de PA para llegar a constatar las horas semanales de realización de dicha práctica.

\section{Resultados}

Para conocer si existe progresión significativa entre los valores del índice de Borg a partir de las respuestas elicitadas por los participantes respecto a los tipos de PA de creciente intensidad, que se ajustan a una distribución normal, y así desarrollar el primer objetivo, se hallaron (Tabla 1) las tendencias correspondientes mediante las ecuaciones de regresión lineal relativas a los tres índices de Borg (Tabla 1), por su carácter predictivo.

Tabla 1. Ecuaciones de regresión lineal correspondientes a las tendencias de índices de Borg de los participantes.

\begin{tabular}{|c|c|c|}
\hline Participantes & Ecuación regresión & $\mathrm{p}$ \\
\hline 1 & $\mathrm{Y}=6,33333+0,0 \mathrm{X}$ & $\mathrm{p}<1$ \\
\hline 2 & $Y=2,66667+1,5 X$ & $\mathrm{p}<0,12$ \\
\hline 3 & $\mathrm{Y}=1+2 \mathrm{X}$ & $\mathrm{p}=1$ \\
\hline 4 & $Y=-0,333333+0,5 X$ & $\mathrm{p}<0,33$ \\
\hline 5 & $Y=1,66667+2,5 X$ & $\mathrm{p}<0,33$ \\
\hline 6 & $\mathrm{Y}=2+1 \mathrm{X}$ & $\mathrm{p}=1$ \\
\hline 7 & $\mathrm{Y}=2+1 \mathrm{X}$ & $\mathrm{p}=1$ \\
\hline 8 & $\mathrm{Y}=3+1 \mathrm{X}$ & $\mathrm{p}=1$ \\
\hline 9 & $Y=0,333333+2 X$ & $\mathrm{p}<0,17$ \\
\hline 10 & $Y=-1,33333+2,5 X$ & $\mathrm{p}<0,07$ \\
\hline 11 & $Y=-0,666667+2,5 X$ & $\mathrm{p}<0,07$ \\
\hline 12 & $Y=-1,33333+3 X$ & $\mathrm{p}<0,12$ \\
\hline 13 & $Y=-2+3 X$ & $\mathrm{p}=1$ \\
\hline 14 & $Y=-0,666667+2,5 X$ & $\mathrm{p}<0,07$ \\
\hline 15 & $Y=-1,66667+2,5 X$ & $\mathrm{p}<0,07$ \\
\hline 16 & $Y=-0,333333+2 X$ & $\mathrm{p}<0,17$ \\
\hline 17 & $Y=-0,666667+2,5 X$ & $\mathrm{p}<0,07$ \\
\hline 18 & $Y=-0,666667+2,5 X$ & $\mathrm{p}<0,07$ \\
\hline 19 & $Y=10,3333-2 X$ & $\mathrm{p}<0,17$ \\
\hline 20 & $Y=4,66667+1,5 X$ & $\mathrm{p}<0,12$ \\
\hline 21 & $\mathrm{Y}=1+2,5 \mathrm{X}$ & $\mathrm{p}<0,21$ \\
\hline 22 & $Y=-0,666667+3 X$ & $\mathrm{p}<0,12$ \\
\hline 23 & $Y=0,333333+2,5 X$ & $\mathrm{p}<0,07$ \\
\hline 24 & $Y=2+2 X$ & $\mathrm{p}=1$ \\
\hline 25 & $\mathrm{Y}=1,66667+2,5 \mathrm{X}$ & $\mathrm{p}<0,07$ \\
\hline 26 & $Y=-0,666667+3 X$ & $\mathrm{p}<0,12$ \\
\hline 27 & $\mathrm{Y}=0,333333+1,5 \mathrm{X}$ & $\mathrm{p}<0,12$ \\
\hline 28 & $Y=-1,66667+3 X$ & $\mathrm{p}<0,12$ \\
\hline 29 & $Y=-2,33333+3 X$ & $\mathrm{p}<0,12$ \\
\hline 30 & $Y=1+2 X$ & $\mathrm{p}=1$ \\
\hline 31 & $Y=-2,66667+3,5 X$ & $\mathrm{p}<0,05$ \\
\hline 32 & $\mathrm{Y}=0+3,5 \mathrm{X}$ & $\mathrm{p}<0,15$ \\
\hline 33 & $Y=-0,666667+2,5 X$ & $\mathrm{p}<0,07$ \\
\hline 34 & $Y=-1,66667+2,5 X$ & $\mathrm{p}<0,07$ \\
\hline 35 & $Y=0,333333+2,5 X$ & $\mathrm{p}<0,33$ \\
\hline
\end{tabular}


Todas las ecuaciones de regresión son ascendentes, y resulta interesante conocer que existe esta progresión, aunque ninguna de ellas es estadísticamente significativa, excepto para el participante 31 .

Por esta razón, para abordar el segundo objetivo y analizar la relación existente entre el IMC de jóvenes deportistas con los valores de FC i RPE, se han promediado los valores de los tres índices de la escala de Borg, así como los de la FC.

Con el fin de hallar la posible existencia de inferencias causales significativas, se propuso un análisis de regresión múltiple, considerando la FC y la RPE como variables predictoras, y el IMC (cociente entre el peso medido en kilogramos y la talla al cuadrado, medida en metros) como variable criterio.

La ecuación de regresión múltiple es la siguiente:

$$
\mathrm{Y}=\beta_{0}+\beta_{1} \mathrm{X}_{1}+\beta_{2} \mathrm{X}_{2}+\mathrm{e}
$$

en la cual Y es la variable criterio IMC, e es la variable aleatoria de error o perturbadora, y las variables $\mathrm{X}_{1} \mathrm{y}_{2}$ son las predictoras, que corresponden, respectivamente, al promedio de la FC y al promedio de los índices de RPE.

La Tabla de análisis de la varianza en la regresión múltiple (Tabla 2) es:

Tabla 2. Tabla de análisis de la varianza en la regresión múltiple.

\begin{tabular}{lccccc}
\hline Fuente & $\begin{array}{c}\text { Suma de } \\
\text { Cuadrados }\end{array}$ & $\begin{array}{c}\text { G.l. } \\
\text { Cuadrado } \\
\text { Medio }\end{array}$ & Razón-F & Valor- $p$ \\
\hline Modelo & 36,2725 & 2 & 18,1362 & 5,32 & 0,0101 \\
Residuo & 109,119 & 32 & 3,40996 & & \\
Total (Corr.) & 145,391 & 34 & & & \\
\hline
\end{tabular}

Dado que el $p$-valor=0,0101 es menor que el nivel de significación $\alpha=0,05$, rechazamos la hipótesis nula; es decir, se cumple que hay relación de dependencia entre las variables predictoras y la variable criterio. El coeficiente de determinación $\mathrm{R}^{2}$ es 0,24948. En consecuencia, el modelo así ajustado permite explicar un $24,948 \%$ del IMC de forma estadísticamente significativa.

\section{Discusión}

En este estudio hemos analizado una intervención de PA a jóvenes deportistas, sometiéndoles a tres cargas de trabajo, acorde al ACSM (2011) y en la que hemos analizado de manera directa la evolución de las variables de la FC y de RPE. En estudios de esta misma línea relativos a los deportes colectivos se hace referencia a la RPE como un factor psicológico de control y éste lo relacionan con otros indicadores de carácter fisiológico como el lactato, consumo de oxígeno y la FC. Estos indicadores también se suelen estudiar para reforzar los valores de la RPE ya que existe un componente subjetivo en la elicitación de la respuesta individual a la escala. En este sentido, Robertson y Noble (1997) encuentran una correlación directa entre la RPE y la FC, al contrario que Crumpton Scharff-Olson, Williford, Bradford y Walker (1999) y Muyor y López (2009), que no hallan una buena relación entre la RPE y la FC en sus estudios de ciclismo indoor, ya que un ejercicio de intensidad media fue percibida como dura (según los parámetros del ACSM (1998): Muy ligera; Ligera; Moderada; Dura; Muy dura y Máxima).

En nuestro estudio, los valores de la RPE son dispares entre ellos en el punto de inicio (positivo, neutro o negativo) y destacamos que en ningún caso la tendencia es estadísticamente significativa, pero sí podemos afirmar que en todos los participantes es ascendente. Esto podría ser explicado por el modo en que se administra la RPE ya que los parámetros que la constituyen son de difícil medición al tratarse de una percepción subjetiva tal y como indica Rodríguez (1995), al modo como ocurre con escalas analógicas que se administran de manera similar, como es la escala analógica visual del dolor (EVA), (Scott y Huskinsson, 1976) que mide de manera subjetiva el dolor que se percibe en una escala del 1 al 10. Cabe destacar que la capacidad perceptiva del esfuerzo se va desarrollando al adquirir mayor adherencia deportiva (Arruza, Alzate y Valencia, 1996) y los jóvenes deportistas de la muestra poseen una cierta adherencia y un conocimiento, en experiencias previas de dicha escala. Dicho conocimiento junto a la fase de observación indirecta que hemos establecido con los participantes en el estudio ha permitido responder a la escala de manera adecuada (Naclerio, et al., 2009). En esta línea, destacamos que los resultados aportados por Robertson y Noble (1997), Little y Williams (2007) y Borrensen y Lambert (2008) coinciden, en cierta manera, con los que hemos obtenido, dado que podemos afirmar que la RPE es creciente a lo largo de las tres actividades planteadas. Así constatamos una cierta correspondencia con estudios referidos al fútbol (Calahorro et al., 2014), al judo (Arruza et al., 1996) y al balonmano (Cuadrado-Reyes et al., 2012). Sin embargo, dichos estudios persiguen su objetivo en las cargas de entrenamiento y en modalidades de deporte distintas. El hallazgo más importante de este estudio se revela a través del modelo de la regresión lineal múltiple que destaca que la cuarta parte del IMC de los jóvenes deportistas se puede explicar por la FC y la RPE. En este sentido, Campos et al. (2012) demostraron que la FC estaba relacionada con el porcentaje de grasa corporal pero no con el volumen de oxígeno máximo, lo que abre nuevas posibilidades de estudio. Los resultados obtenidos en nuestro estudio nos permiten afirmar que, en un $24,948 \%$, tenemos la garantía de que aquellos participantes que tienen los valores de FC y de la RPE más altos también tienen los valores de IMC más altos. 


\section{Aplicaciones prácticas}

Siguiendo con la línea práctica de la discusión, el concepto RPE de sesión se manifiesta como una aplicación fiable para el control de la fatiga y eso ha permitido seguir avanzando en este campo y en el del entrenamiento deportivo (Cuadrado-Reyes et al., 2012). Una limitación a tener en cuenta para administrar la escala de Borg es la percepción real del participante ya que diversos trabajos (Naclerio et al, 2009) sugieren la necesidad de familiarizarse con la escala y realizar un tiempo de aprendizaje de 8 semanas. En nuestro estudio no se ha llevado a cabo dicho tiempo de aprendizaje ya que se consideró que los alumnos de Ciencias de la Actividad Física y el Deporte conocían y entendían perfectamente el concepto de esfuerzo percibido asimismo de haberlo reforzado mediante la fase de observación indirecta.
Becas, ayudas o soporte financiero: Este articulo se ha realizado con el apoyo y financiación de: Grup de recerca $i$ innovació en dissenys (GRID). Tecnologia $i$ aplicació multimedia $i$ digital als dissenys observacionals. Grupo Consolidado de la Generalitat de Catalunya [Referencia: 2014 SGR 971]. Asimismo, ha recibido el apoyo del Proyecto I+D+I: Observación de la interacciónen deporte y actividad física: Avances técnicos $y$ metodológicos en registros automatizados cualitativos-cuantitativos. Secretaria de Estado de Investigación, Desarrollo e Innovación del Ministerio de Economía y Competitividad [Referencia: DEP2012-32124] y del proyecto INEFCP 2012: Programes de motricitat perceptiva i expressiva en bordelines $i$ gent gran. Beneficis i creació d'intruments ad hoc.

\section{Referencias}

1. Ainsworth BE, Haskell WL, Whitt MC, Irwin ML, Swartz AM, Strath SJ, O’Brien WL, Bassett DR Jr, Schmitz KH, Emplaincourt PO, Jacobs DR Jr, Leon AS. Compendium of Physical Activities: An update of activity codes and MET intensities. Medicine and Science in Sports and Exercise, 2000,32 (Suppl),S498-S516.

2. American College of Sports Medicine (ACSM) (1998). ACSM position stand on the recommended quantity and quality of exercise for developing and maintaining cardiorespiratory and muscular fitness, and flexibility in adults. Medicine \& Science in Sports \& Exercise,30(6), 975-91.

3. American College of Sport Medicine. (2011). American College of Sports Medicine position stand. Quantity and quality of exercise for developing and maintaining cardiorespiratory, musculoskeletal, and neuromotor fitness in apparently healthy adults: guidance for prescribing exercise. Jul,43(7),1334-59.

4. Alexiou, H. y Coutts, A. (2008). A comparison of methods used for quantifying internal training load in women soccer players. International Journal of Sports, Physiology and Performance, 3, 320-330.

5. Anguera, M.T., Camerino, O., Castañer, M. y Sánchez-Algarra, P. (2014). Mixed methods en actividad física y deporte. Revista de Psicología del Deporte, 23 (1), 123-130.

6. Arruza, J., Alzate, R. y Valencia, J. (1996). Esfuerzo percibido y frecuencia cardiaca: El control de la intensidad de los esfuerzos en el entrenamiento de judo. Revista de Psicología del Deporte, 5(2), 29-40.

7. Bar-Or, O., Skinner, J.S., Bruskirk, E.R. y Borg, G. (1972). Physiological and perceptual indicators of physical stress in 41 to 60 year old men who vary in conditioning level and in body fatness. Medicine and Science in Sports, 4, 96-100.

8. Blair, S. y Morris, J. (2009). Healthy Hearts and the universal benefits of being physically active: physical activity and health. Annals of Epidemiology, 19(4), 253-256.

9. Borg, G. (1962). A simple rating scale for use in physical work test. Fysiografiska Sällskapets Lund Förhandlingar, 32, 7-15.

10. Borg, G. (1973). Perceived exertion: a note on "history" and methods. Medicine and Science in Sports, 5, 90-93.

11. Borg, G. (1982). A category scale with ratio properties for intermodal and interindividual comparisons. En: H. G. Geissler y P. Petzold (Ed.), Psychophysical judgment and the process of perception (pp. 25-34). Berlín VEB Deutscher Verlagder Wissenschaften.

12. Borg, G. y Linderholm, H. (1970). Exercise performance and perceived exertion in patients with coronary insufficiency, arterial hypertension, and vasoregulatory asthenia. Acta Medica Scandinavica, 187, 17-26.

13. Borresen, J. y Lambert, M. I. (2008). Quantifying training load: A comparison of subjective and objective methods. International Journal of Sports, Physiology \& Performance, 3 (1), 16-30.

14. Brownson, R.C., Boehmer, T.K. y Luke, D.A. (2005). Declining rates of physical activity in the United States: What are the contributors? Annual Review of Public Health, 26, 421-443.

15. Calahorro, F., Torres, G. y Lara, A.J. (2014). La percepción subjetiva de esfuerzo como herramienta válida para la monitorización de la intensidad del esfuerzo en competición de jóvenes futbolistas. Cuadernos de Psicología del Deporte, 14(1), 75-82.

16. Campos, .Z., Bastos, N., Papoti, M., Freitas, F., Gobatto, A. y Balikian, P (2012). The effects of physical fitness and body composition on oxygen consumption and heart rate recovery after high-intensity exercise. Internacional Journal Sports of Medicine, 33, 621-626

17. Casamichana, D., Castellano, J. y Blanco-Villaseñor, A. (2012). Estudio de la percepción subjetiva del esfuerzo en tareas de entrenamiento en fútbol a través de la teoría de la generalizabilidad. Revista de Psicología del Deporte, 21(1), 35-40.

18. Castañer, M. y Camerino, O. (2006): Manifestaciones Básicas de la Motricidad. Lleida: INEFC. Publicacions de la Universitat de Lleida.

19. Castañer, M. y Saüch, G. (2014). Patterns of motor behaviour in the elderly. Procedia - Social and Behavioral Sciences 116, 2074 - 2077.

20. Castañer, M., Torrents, C., Anguera, M.T., Dinušová, M., y Jonsson, G.K. (2009): Identifying and analyzing motor skill responses in body movement and dance. Behavior Research Methods. 41 (3), 857-867.

21. Chen, M. J., Fan, X. y Moe, S. T. (2002). Criterion-related validity of the Borg ratings of perceived exertion scale in healthy individuals: a meta-analysis. Journal of Sports Sciences, 20(11), 873-899.

22. Coutts, A., Reaburn, P., Abt, G. (2003). Heart rate, blood lactate concentration and estimated energy expenditure in a semi-professional rugby league team during a match: a case study. Journal of Sports Sciences, 21, 97-103.

23. Cuadrado-Reyes, J., Chirosa, L.J., Chirosa, I., Martín, I. y Aguilar, D. (2012). La percepción subjetiva del esfuerzo para el control de la carga entrenamiento en una temporada en un equipo de balonmano. Revista de Psicología del Deporte, 21(2), 331-339.

24. Crumpton, S., Scharff-Olson, M., Williford, H. N., Bradford, A. y Walker, S. (1999). The effects of a commercially-produced "Spinning" video: aerobic responses and caloric expenditure. Medicine \& Science in Sports \& Exercise, 31(5), 112. 
25. Dreyfus, H. L. y Dreyfus. S. E. (1986). Mind over machine. The power of human intuition and expertise in the era of the computer. New York: The Free Press.

26. Fang J, Wylie-Rosett J, Cohen HW, Kaplan RC, y Alderman MH. (2003). Exercise, body mass index, caloric intake, and cardiovascular mortality. American Journal of Preventive Medicine, 25, 283-289.

27. Fernández - Castanys, B. F., Chirosa, L. J. y Chirosa, I. (2002). Validez del uso de la RPE en el control de la intensidad de entrenamiento en balonmano. Archivos de Medicina del Deporte, 91, 377-383.

28. Genberg, V. (1992): Patterns and Organizing Perspectives: a View of Expertise. Teaching and Teacher Education, 8(5/6), 485-495.

29. Guerra, I., Chaves, R., Barros, T. y Tirapegui, J. (2004). The Influence of Fluid Ingestion on Performance of Soccer Players during a Match. Journal of Sports Science and Medicine, 3, 198-202.

30. Gomez-Piriz, P. T., Jiménez-Reyes, P. y Ruiz-Ruiz, C. (2011). Relation Between Total Body Load and Session Rating of Perceived Exertion in Professional Soccer Players. Journal of Strength and Conditioning Research, 25, 2100-2103.

31. Hartwig, T., Naughton, G. y Searl, J. (2008). Defining the volume and intensity of sport participation in adolescent rugby union players. International Journal of Sports Physiology and Performance, 3, 94-106.

32. Impellizzeri, F., Rampinini, E., Coutts, A., Sassi, A. y Marcora, S. (2004). Use of RPE-based training load in soccer. Medicine and Science in sports and Exercise, 36, 1042-1047.

33. Lagally K. M., McGaw, S.T., Young, G.T., Heather, C. M., y Thomas, D. Q. (2004). Rating of perceived Exertion and Muscle Activity During The Bench Press Exercise in Recreational And Novice Lifters. Journa of Strength and Conditioning, 18(2), 359-364.
34. Little, T. y Williams, A. (2007). Measures of exercise intensity during soccer training drills with professional soccer players. Journal of Strength and Conditioning Research, 21(2), 367-371.

35. Muyor, J.M. y López, P.A. (2009). Respuesta de la frecuencia cardíaca y percepción subjetiva del esfuerzo en principiantes, durante la práctica de ciclismo indoor. Motricidad. European Journal of Human Movement, 23, 49-57.

36. Naclerio, F., Barriopedro, I. y Rodríguez, G. (2009). Control de la Intensidad en los Entrenamientos de Fuerza por medio de la Percepción Subjetiva del Esfuerzo. Kronos, 8(14), 59-66.

37. Plasqui, G. y Westerterp, K.R. (2007). Physical activity assessment with accelerometers: an evaluation against doubly labeled water. Obesity, 15(10), 2371-9.

38. Robertson, R. J. y Noble, B. N. (1997). Perception of physical exertion: methods, mediators, and applications (pp. 407-452). En Holloszy, J. O. (Ed.). Exercise and Sport Sciences Review. Baltimore: Williams \& Williams.

39. Rodríguez, F. A. (1995). Prescripción del ejercicio físico para la salud. Resistencia cardiorrespiratoria. Apunts: Educación Física y Deportes, 39, 87-102.

40. Saüch, G y Castañer, M. (2014): Observación de patrones motrices generados por los programas de actividad física para la tercera edad y la percepción de sus usuarios. Revista de Psicología del Deporte 23 (1), $181-190$

41. Seefeldt V, Malina RM, y Clark MA. (2002). Factors affecting levels of physical activity in adults. Sports Medicine, 32, 143-68.

42. Scott, J., y Huskinsson E.C. (1976). Graphic representation of pain. Pain, 2, 175-84. 